\title{
Existence and Uniqueness of Positive Solution for Third-Order Three-Point Boundary Value Problems
}

\author{
Tongchun Hu${ }^{1}$, Yongping Sun ${ }^{2 *}$ \\ ${ }^{1}$ Department of Public Teaching, Hangzhou Polytechnic, Hangzhou, China \\ ${ }^{2}$ College of Electron and Information, Zhejiang University of Media and Communications, Hangzhou, China \\ Email: hutongchun888@126.com, sunyongping@126.com
}

Received 22 March 2014; revised 14 April 2014; accepted 1 May 2014

Copyright $@ 2014$ by authors and Scientific Research Publishing Inc.

This work is licensed under the Creative Commons Attribution International License (CC BY).

http://creativecommons.org/licenses/by/4.0/

(c) (i) Open Access

\section{Abstract}

This paper is devoted to the study of the existence and uniqueness of the positive solution for a type of the nonlinear third-order three-point boundary value problem. Our results are based on an iterative method and the Leray-Schauder fixed point theorem.

\section{Keywords}

Positive Solution, Uniqueness and Existence, Third-Order Three-Point BVPs

\section{Introduction}

In this paper, we consider the uniqueness and existence of the positive solution for the following third-order differential equation

$$
u^{\prime \prime \prime}(t)+f(t, u(t))=0, \quad t \in(0,1)
$$

or

$$
u^{\prime \prime \prime}(t)+g\left(t, u(t), u^{\prime}(t)\right)=0, \quad t \in(0,1),
$$

with the following three-point boundary conditions

$$
u(0)=u^{\prime}(0)=0, \quad u^{\prime}(1)=a u^{\prime}(\eta) .
$$

Throughout this paper, we assume that $\eta \in(0,1), a \in(0,1 / \eta), f \in C((0,1) \times[0, \infty),[0, \infty))$ may be singular ${ }^{*}$ Corresponding author. 
at $t=0$ and/or $t=1$ and $g \in C([0,1] \times[0, \infty) \times[0, \infty),[0, \infty))$. Here, the solution $u^{*}(t)$ of the BVP (1)-(3) (or the BVP (2)-(3)) is called positive if $u^{*}(t)>0, t \in(0,1)$.

In the past few years, because of the extensive applications in mechanics and engineering, the existence of solutions or positive solutions for nonlinear singular or nonsingular three-point boundary value problems for third-order ordinary differential equations has been studied extensively in the literature (see [1]-[13] and references therein). For example, in the case of $a \in(1,1 / \eta)$ and $f(t, u)$ is nonsingular at $t=0$ and $t=1$, Guo $e t$ al. [1] [2] established some existence results of at least one and at least three positive solutions for the BVP (1)(3) by using the well-known Krasnosel'skii fixed point theorem and the Leggett-Williams fixed point theorem, respectively. By using the upper and lower solutions and the maximum principle, Yao and Feng in [14] and Feng and Liu in [15] studied the existence of solutions for the BVP (1)-(3) and BVP (2)-(3) with $a=0$, respectively.

Motivated mainly by the papers mentioned above, in this paper we will consider the uniqueness of the positive solution, the iteration and the rate of the convergence by the iteration for the nonlinear singular third-order three-point BVP (1)-(3). We study the existence of the positive solution for the nonlinear third-order three-point BVP (2)-(3) by using the Leray-Schauder fixed point theorem.

The rest of this paper is organized as follows. After this section, we present some notations and lemmas that will be used to prove our main results in Section 2. We discuss the uniqueness in Section 3. Finally, we discuss the existence in Section 4.

\section{Preliminaries}

In this section, we introduce definitions and preliminary facts which are used throughout this paper.

Definition 1 Let $E$ be a real Banach space. A nonempty closed convex set $K \subset E$ is called a cone of $E$ if it satisfies the following two conditions:

1) $x \in K, \lambda \geq 0$ implies $\lambda x \in K$;

2) $x \in K,-x \in K$ implies $x=0$.

Definition 2 An operator is called completely continuous if it is continuous and maps bounded sets into precompact sets.

The following lemma plays a pivotal role in the forthcoming analysis.

Lemma 3 [9] Suppose that $a \eta \neq 1, h \in C[0,1]$, then the unique solution of the following equation

$$
u^{\prime \prime \prime}(t)+h(t)=0, \quad t \in(0,1)
$$

with boundary conditions (3) is given by

$$
u(t)=\int_{0}^{1} G(t, s) h(s) \mathrm{d} s,
$$

where

$$
\begin{gathered}
G(t, s)=K(t, s)+\frac{a t^{2}}{2(1-a \eta)} K_{1}(\eta, s), \\
K(t, s)=\frac{1}{2} \begin{cases}\left(2 t-t^{2}-s\right) s, & 0 \leq s \leq t \leq 1, \\
(1-s) t^{2}, & 0 \leq t \leq s \leq 1,\end{cases}
\end{gathered}
$$

and

$$
K_{1}(t, s):=\frac{\partial K(t, s)}{\partial t}= \begin{cases}(1-t) s, & 0 \leq s \leq t \leq 1 \\ (1-s) t, & 0 \leq t \leq s \leq 1 .\end{cases}
$$

We need some properties of functions $K(t, s), K_{1}(t, s)$ and $G(t, s)$ in order to establish the existence and uniqueness of positive solutions.

Lemma 4 For all $(t, s) \in[0,1] \times[0,1]$, we have

$$
0 \leq K_{1}(t, s) \leq t .
$$

Proof The conclusion is obvious. The proof is completed. 
Lemma 5 For all $(t, s) \in[0,1] \times[0,1]$, we have

$$
0 \leq K(t, s) \leq t^{2} .
$$

Proof For all $t, s \in[0,1]$, if $s \leq t$, it follows from (7) that

$$
K(t, s)=\frac{1}{2}\left(2 t-t^{2}-s\right) s \leq \frac{1}{2}\left(2 t-t^{2}\right) t \leq t^{2},
$$

and

$$
K(t, s)=\frac{1}{2}\left(2 t-t^{2}-s\right) s=\frac{1}{2}[(1-t) t+(t-s)] s \geq 0 .
$$

If $t \leq s$, then from (7) we have

$$
0 \leq K(t, s)=\frac{1}{2} t^{2}(1-s) \leq t^{2}
$$

The proof is completed.

Lemma 6 The Green's function $G(t, s)$ has the following properties:

$$
\begin{aligned}
& \max _{0 \leq t \leq 1} \int_{0}^{1} G(t, s) \mathrm{d} s=\frac{1+2 a \eta-3 a \eta^{2}}{12(1-a \eta)}, \\
& \max _{0 \leq t \leq 1} \int_{0}^{1} \frac{\partial}{\partial t} G(t, s) \mathrm{d} s=M= \begin{cases}\frac{1-a \eta^{2}}{4(1-a \eta)}, & a \leq \frac{1}{\eta(2-\eta)}, \\
\frac{a \eta(1-\eta)}{2(1-a \eta)}, & a \geq \frac{1}{\eta(2-\eta)} .\end{cases}
\end{aligned}
$$

Proof After direct computations, we easily get

$$
\begin{aligned}
& \int_{0}^{1} G(t, s) \mathrm{d} s=-\frac{1}{6} t^{3}+\frac{1-a \eta^{2}}{4(1-a \eta)} t^{2}, \\
& \int_{0}^{1} \frac{\partial}{\partial t} G(t, s) \mathrm{d} s=-\frac{1}{2} t^{2}+\frac{1-a \eta^{2}}{2(1-a \eta)} t .
\end{aligned}
$$

From (11) and (12) we can get (9) and (10) respectively. The proof is completed.

\section{Uniqueness}

We shall consider the Banach space $E=C[0,1]$ equipped with norm $\|u\|=\max _{0 \leq t \leq 1}|u(t)|$.

Theorem 7 Suppose that

(H1) $f\left(t, u_{1}\right) \leq f\left(t, u_{2}\right)$ for any $0<t<1,0 \leq u_{1} \leq u_{2}$;

(H2) There exist $q \in(0,1)$ such that

$$
f(t, r x) \geq r^{q} f(t, x), \text { for any } r \in(0,1),(t, x) \in(0,1) \times[0, \infty) ;
$$

(H3) $0<\int_{0}^{1} f\left(s, s^{2}\right) \mathrm{d} s<\infty$.

Then the BVP (1)-(3) has an unique positive, nondecreasing solution $u^{*} \in D \cap C^{3}(0,1)$, here

$$
D=\left\{x \in C[0,1] \mid \exists M_{x} \geq m_{x} \geq 0 \text {, such that } m_{x} t^{2} \leq x(t) \leq M_{x} t^{2}, t \in[0,1] .\right\} .
$$

Constructing successively the sequence of functions

$$
h_{n}(t)=\int_{0}^{1} G(t, s) f\left(s, h_{n-1}(s)\right) \mathrm{d} s, t \in[0,1], n=1,2, \cdots,
$$

for any initial function $h_{0}(t) \in D$, then $\left\{h_{n}(t)\right\}$ must converge to $u^{*}(t)$ uniformly on $[0,1]$ and the rate of convergence is 


$$
\max _{t \in[0,1]}\left|h_{n}(t)-u^{*}(t)\right|=O\left(1-\theta^{q^{n}}\right) .
$$

where $0<\theta<1$, which depends on the initial function $h_{0}(t)$.

Proof Obviously, from (H1) we obtain

$$
f(t, \lambda x) \leq \lambda^{q} f(t, x), \quad \forall \lambda>1,(t, x) \in((0,1) \times[0, \infty)) .
$$

Let

$$
C^{+}[0,1]=\{u \in E \mid u(t) \geq 0, t \in[0,1]\} .
$$

In view of Lemma 3, we define an operator $T$ as

$$
(T u)(t)=\int_{0}^{1} K(t, s) f(s, u(s)) \mathrm{d} s+\frac{\alpha t^{2}}{2(1-\alpha \eta)} \int_{0}^{1} K_{1}(\eta, s) f(s, u(s)) \mathrm{d} s, u \in D .
$$

By (H1) it is easy to see that the operator $T: D \rightarrow C^{+}[0,1]$ is increasing. Observe that the BVP (1)-(3) has a solution if and only if the operator $T$ has a fixed point.

In what follows, we first prove $T: D \rightarrow D$. In fact, for any $u \in D$, there exist positive numbers $0<m_{u}<1<M_{u}$ such that

$$
m_{u} s^{2} \leq u(s) \leq M_{u} s^{2}, \quad s \in[0,1]
$$

It follows from (H2) and (16) that

$$
\left(m_{u}\right)^{q} f\left(s, s^{2}\right) \leq f(s, u(s)) \leq\left(M_{u}\right)^{q} f\left(s, s^{2}\right), \quad s \in(0,1) .
$$

Using (17), (18), (8) and the condition (H1), we obtain

$$
\begin{aligned}
(T u)(t) & =\int_{0}^{1} K(t, s) f(s, u(s)) \mathrm{d} s+\frac{\alpha t^{2}}{2(1-\alpha \eta)} \int_{0}^{1} K_{1}(\eta, s) f(s, u(s)) \mathrm{d} s \\
& \leq t^{2} \int_{0}^{1} f(s, u(s)) \mathrm{d} s+\frac{\alpha t^{2}}{2(1-\alpha \eta)} \int_{0}^{1} K_{1}(\eta, s) f(s, u(s)) \mathrm{d} s \\
& \leq t^{2} \int_{0}^{1}\left(M_{u}\right)^{q} f\left(s, s^{2}\right) \mathrm{d} s+\frac{\alpha t^{2}}{2(1-\alpha \eta)} \int_{0}^{1} K_{1}(\eta, s)\left(M_{u}\right)^{q} f\left(s, s^{2}\right) \mathrm{d} s \\
& =\left(\left(M_{u}\right)^{q} \int_{0}^{1} f\left(s, s^{2}\right) \mathrm{d} s+\frac{\alpha\left(M_{u}\right)^{q}}{2(1-\alpha \eta)} \int_{0}^{1} K_{1}(\eta, s) f\left(s, s^{2}\right) \mathrm{d} s\right) t^{2}, \quad t \in[0,1],
\end{aligned}
$$

and

$$
\begin{aligned}
(T u)(t) & =\int_{0}^{1} K(t, s) f(s, u(s)) \mathrm{d} s+\frac{\alpha t^{2}}{2(1-\alpha \eta)} \int_{0}^{1} K_{1}(\eta, s) f(s, u(s)) \mathrm{d} s \\
& \geq \frac{\alpha t^{2}}{2(1-\alpha \eta)} \int_{0}^{1} K_{1}(\eta, s) f(s, u(s)) \mathrm{d} s \\
& \geq \frac{\alpha t^{2}}{2(1-\alpha \eta)} \int_{0}^{1} K_{1}(\eta, s)\left(m_{u}\right)^{q} f\left(s, s^{2}\right) \mathrm{d} s \\
& =\left(\frac{\alpha\left(m_{u}\right)^{q}}{2(1-\alpha \eta)} \int_{0}^{1} K_{1}(\eta, s) f\left(s, s^{2}\right) \mathrm{d} s\right) t^{2}, \quad t \in[0,1] .
\end{aligned}
$$

Equations (19), (20) and (H5) imply that $T: D \rightarrow D$.

For any $h_{0} \in D$, we let 


$$
\begin{aligned}
& l_{h_{0}}=\sup \left\{l>0: l h_{0}(t) \leq\left(T h_{0}\right)(t), t \in[0,1]\right\}, \\
& L_{h_{0}}=\inf \left\{L>0:\left(T h_{0}\right)(t) \leq L h_{0}(t), t \in[0,1]\right\}, \\
& m=\min \left\{1,\left(l_{h_{0}}\right)^{\frac{1}{1-q}}\right\}, \quad M=\max \left\{1,\left(L_{h_{0}}\right)^{\frac{1}{1-q}}\right\},
\end{aligned}
$$

and

$$
\begin{aligned}
& u_{0}(t)=m h_{0}(t), \quad v_{0}(t)=M h_{0}(t), \\
& u_{n}(t)=T u_{n-1}(t), \quad v_{n}(t)=T v_{n-1}(t), \quad n=0,1,2, \cdots .
\end{aligned}
$$

Since the operator $T$ is increasing, (H1), (H2), (21) and (22) imply that

$$
u_{0}(t) \leq u_{1}(t) \leq \cdots \leq u_{n}(t) \leq \cdots \leq v_{n}(t) \leq \cdots \leq v_{1}(t) \leq v_{0}(t), \quad t \in I .
$$

For $\theta=m / M$, from (H1), (17) and (22), it can obtained by induction that

$$
u_{n}(t) \geq \theta^{q^{n}} v_{n}(t), \quad t \in[0,1], n=0,1,2, \cdots .
$$

From (23) and (24) we know that

$$
0 \leq u_{n+p}(t)-u_{n}(t) \leq v_{n}(t)-u_{n}(t) \leq\left(1-\theta^{q^{n}}\right) M h_{0}(t), \quad \forall n, p \in \mathbb{N},
$$

so that there exists a function $u^{*}(t) \in D$ such that

$$
u_{n}(t) \rightarrow u^{*}(t), \quad v_{n}(t) \rightarrow u^{*}(t), \quad(\text { uniformly on }[0,1]),
$$

and

$$
u_{n}(t) \leq u^{*}(t) \leq v_{n}(t), \quad t \in[0,1], n=0,1,2, \cdots .
$$

From (H1) and (22) we have

$$
u_{n+1}(t)=T u_{n}(t) \leq T u^{*}(t) \leq T v_{n}(t)=v_{n+1}(t), \quad n=0,1,2, \cdots .
$$

This together with (26) and uniqueness of the limit imply that $u^{*}$ satisfy $u^{*}=T u^{*}$, thus $u^{*} \in C[0,1] \cap C^{3}(0,1)$ is a solution of the BVP (1)-(3).

Form (22), (23) and (H1), we obtain

$$
u_{n}(t) \leq h_{n}(t) \leq v_{n}(t), \quad t \in[0,1], n=0,1,2, \cdots .
$$

It follows from (26), (27) and (28) that

$$
\left|h_{n}(t)-u^{*}(t)\right| \& \leq\left|h_{n}(t)-u_{n}(t)\right|+\left|u_{n}(t)-u^{*}(t)\right| \leq 2\left|v_{n}(t)-u_{n}(t)\right| \leq 2\left(1-\theta^{q^{n}}\right) M\left|h_{0}(t)\right| .
$$

Therefore,

$$
\max _{t \in[0,1]}\left|h_{n}(t)-u^{*}(t)\right| \leq 2\left(1-\theta^{q^{n}}\right) M \max _{t \in[0,1]}\left|h_{0}(t)\right| .
$$

So that (15) holds. Since $h_{0}(t)$ is arbitrary in $D$ we know that $u^{*}(t)$ is the unique solution of the BVP (1)-(3) in $D$.

Remark If $f(t, u)$ is continuous on $[0,1] \times[0, \infty)$, then it is quite evident that the condition (H3) holds. Hence the unique solution $u^{*}(t)$ is in $C^{3}[0,1]$.

\section{Existence}

Now we are ready to discuss the existence of positive solutions for the BVP (2)-(3).

Theorem 8 Suppose that

(H4) $g \in C([0,1] \times[0, \infty) \times[0, \infty),[0, \infty))$ and $g(t, 0,0) \not \equiv 0, t \in[0,1]$;

(H5) There exists positive number $d>0$ such that 


$$
\max \left\{g\left(t, u_{0}, u_{1}\right):\left(t, u_{0}, u_{1}\right) \in[0,1] \times[0, d] \times\left[0, \frac{12(1-a \eta) M}{1+2 a \eta-3 a \eta^{2}} d\right]\right\} \leq \frac{12(1-a \eta)}{1+2 a \eta-3 a \eta^{2}} d,
$$

where $M$ is defined by (11).

Then the BVP (2)-(3) has at least one positive solution $u^{*}(t)$ such that

$$
0 \leq u^{*}(t) \leq d, \quad 0 \leq\left(u^{*}\right)^{\prime}(t) \leq \frac{12(1-a \eta) M}{1+2 a \eta-3 a \eta^{2}} d, \quad t \in[0,1] .
$$

Proof We consider the Banach space $E=C^{1}[0,1]$ equipped with the norm

$$
\|u\|=\max \left\{|u|_{0}, \frac{1+2 a \eta-3 a \eta^{2}}{12(1-a \eta) M}\left|u^{\prime}\right|_{0}\right\},
$$

where $|u|_{0}=\max _{0 \leq t \leq 1}|u(t)|$.

For $u \in E$, define the operator $S$ by

$$
(S u)(t)=\int_{0}^{1} G(t, s) g\left(s, u(s), u^{\prime}(s)\right) \mathrm{d} s, \quad t \in[0,1] .
$$

By Ascoli-Arzela Theorem, it is easy to known that the operator $S: E \rightarrow E$ is a completely continuous operator. The BVP (2)-(3) has a solution $u=u(t)$ if and only if $u$ is a fixed point of operator $S$ defined by (32). Let

$$
\Omega_{d}=\left\{u \in E:\|u\|<d, u(t) \geq 0, u^{\prime}(t) \geq 0, t \in[0,1]\right\},
$$

then $\Omega_{d}$ is a bounded closed convex set of $E$. We show that $T\left(\Omega_{d}\right) \subseteq \Omega_{d}$. For $u \in \Omega_{d}$, by (31) we have

$$
|u|_{0} \leq d,\left|u^{\prime}\right|_{0} \leq \frac{12(1-a \eta) M}{1+2 a \eta-3 a \eta^{2}} d,
$$

which implies that

$$
0 \leq u(t) \leq d, 0 \leq u^{\prime}(t) \leq \frac{12(1-a \eta) M}{1+2 a \eta-3 a \eta^{2}} d, \quad t \in[0,1] .
$$

Therefore, by (9), (10), (29) and (32) we get

$$
\begin{aligned}
|S u|_{0} & =\max _{0 \leq t \leq 1}\left|\int_{0}^{1} G(t, s) g\left(s, u(s), u^{\prime}(s)\right) \mathrm{d} s\right| \\
& =\max _{0 \leq t \leq 1} \int_{0}^{1} G(t, s) g\left(s, u(s), u^{\prime}(s)\right) \mathrm{d} s \\
& \leq \frac{12(1-a \eta)}{1+2 a \eta-3 a \eta^{2}} d \max _{0 \leq t \leq 1} \int_{0}^{1} G(t, s) \mathrm{d} s=d,
\end{aligned}
$$

and

$$
\begin{aligned}
\left|(S u)^{\prime}\right|_{0} & =\max _{0 \leq t \leq 1}\left|\int_{0}^{1} \frac{\partial}{\partial t} G(t, s) g\left(s, u(s), u^{\prime}(s)\right) \mathrm{d} s\right| \\
& =\max _{0 \leq t \leq 1} \int_{0}^{1} \frac{\partial}{\partial t} G(t, s) g\left(s, u(s), u^{\prime}(s)\right) \mathrm{d} s \\
& \leq \frac{121-a \eta}{1+2 a \eta-3 a \eta^{2}} d \max _{0 \leq t \leq 1} \int_{0}^{1} \frac{\partial}{\partial t} G(t, s) \mathrm{d} s=\frac{12(1-a \eta) M}{1+2 a \eta-3 a \eta^{2}} d .
\end{aligned}
$$

Then (33) and (34) show that

$$
\|S u\|=\max \left\{|S u|_{0}, \frac{1+2 a \eta-3 a \eta^{2}}{12(1-a \eta) M}\left|(S u)^{\prime}\right|_{0}\right\} \leq d .
$$

i.e., $S u \in \Omega_{d}$. Thus, by Leray-Schauder fixed point theorem, $S$ has a fixed point $u^{*} \in \Omega_{d}$, which implies that 
BVP (2)-(3) has at least one positive solution $u^{*}$ satisfying (30). This completes the proof.

\section{Acknowledgements}

The authors thank the referee for her/his careful reading of the paper and useful suggestions. This work is supported by Hangzhou Polytechnic (KZYZ-2009-2) and the Natural Science Foundation of Zhejiang Province of China (LY12A01012).

\section{References}

[1] Guo, L., Sun, J. and Zhao, Y. (2007) Multiple Positive Solutions for Nonlinear Third-Order Three-Point Boundary-Value Problems. Electronic Journal of Differential Equations, 112, 1-7.

[2] Guo, L., Sun, J. and Zhao, Y. (2008) Existence of Positive Solution for Nonlinear Third-Order Three-Point Boundary Value Problem. Nonlinear Analysis, Theory, Methods and Applications, 68, 3151-3158.

[3] Anderson, D. (1998) Multiple Positive Solutions for a Three-Point Boundary Value Problem. Mathematical and Computer Modelling, 27, 49-57. http://dx.doi.org/10.1016/S0895-7177(98)00028-4

[4] Graef, J.R. and Yang, B. (2005) Multiple Positive Silutions to a Three Point Third Order Boundary Value Problem. Discrete and Continuous Dynmical Systems, 1-8.

[5] Palamides, P.K. and Palamides, A.P. (2008) A Third-Order 3-Point BVP. Applying Krasnosel'skii's Theorem on the Plane without a Green's Function. Electronic Journal of Differential Equations, 14, 1-15.

[6] Palamides, A.P. and Stavrakakis, N.M. (2010) Existence and Uniqueness of a Positive Solution for a Third-Order Three-Point Boundary-Value Problem. Electronic Journal of Differential Equations, 155, 1-12.

[7] Sun, J., Ren, Q. and Zhao, Y. (2010) The Upper and Lower Solution Method for Nonlinear Third-Order Three-Point Boundary Value Problem. Electronic Journal of Qualitative Theory of Differential Equations, 26, 1-8.

[8] Sun, Y. (2005) Positive Solutions of Singular Third-Order Three-Point Boundary Value Problems. Journal of Mathematical Analysis and Applications, 306, 589-603. http://dx.doi.org/10.1016/j.jmaa.2004.10.029

[9] Sun, Y. (2009) Positive Solutions for Third-Order Three-Point Nonhomogeneous Boundary Value Problems. Applied Mathematics Letters, 22, 45-51. http://dx.doi.org/10.1016/j.aml.2008.02.002

[10] Sun, Y. (2008) Existence of Triple Positive Solutions for a Third-Order Three-Point Boundary Value Problem. Journal of Computational and Applied Mathematics, 221, 194-201. http://dx.doi.org/10.1016/j.cam.2007.10.064

[11] Torres, F.J. (2013) Positive Solutions for a Third-Order Three-Point Boundary-Value Problem. Electronic Journal of Differential Equations, 147, 1-11.

[12] Yao, Q. (2009) Positive Solutions of Singular Third-Order Three-Point Boundary Value Problems. Journal of Mathematical Analysis and Applications, 354, 207-212. http://dx.doi.org/10.1016/j.jmaa.2008.12.057

[13] Zhang, X. and Liu, L. (2008) Nontrivial Solution of Third-Order Nonlinear Eigenvalue Problems (II). Applied Mathematics and Computation, 204, 508-512. http://dx.doi.org/10.1016/j.amc.2008.06.048

[14] Yao, Q. and Feng, Y. (2002) The Existence of Solutions for a Third Order Two-Point Boundary Value Problem. Applied Mathematics Letters, 15, 227-232. http://dx.doi.org/10.1016/S0893-9659(01)00122-7

[15] Feng, Y. and Liu, S. (2005) Solvability of a Third-Order Two-Point Boundary Value Problem. Applied Mathematics Letters, 18, 1034-1040. http://dx.doi.org/10.1016/j.aml.2004.04.016 